\title{
Clq nephropathy among children with nephrotic syndrome: Ten-year experience from a pediatric nephrology unit
}

\author{
Nisreen Abu-Shahin ${ }^{1}$, Ali Al-Khader ${ }^{1}$, Duaa' Qattan ${ }^{1}$ Kamal Akl ${ }^{2}$, \\ Departments of ${ }^{1}$ Pathology and ${ }^{2}$ Pediatrics, University of Jordan, Faculty of Medicine, Amman, Jordan. \\ E-mail: nisreenshahin@yahoo.com \\ Received: 12th March 2017, Revised: 1st August 2017, Accepted: 23th September 2017
}

SUMMARY: Abu-Shahin N, Al-Khader A, Qattan D, Akl K. Clq nephropathy among children with nephrotic syndrome: Ten-year experience from a pediatric nephrology unit. Turk J Pediatr 2018; 60: 14-21.

$\mathrm{Clq}$ nephropathy $(\mathrm{ClqN})$ is a rare glomerulopathy mostly seen in children, and presents with nephrotic syndrome (NS). Diagnosis depends on immunoflourescence or immunohistochemical C1q mesangial deposition, excluding other immune-mediated diseases. We retrospectively investigated ClqN incidence, clinicopathological features, and outcome among pediatric NS in our institution.Clinical data, microscopic slides and corresponding tissue blocks of pediatric renal biopsies were retrieved. According to diagnostic criteria for C1qN, 53 pediatric NS renal biopsies were selected for AntiClqA IHC stain microscopic examination. Clinicopathological features and follow up data were recorded. ClqN incidence was $9.4 \%$ among pediatric NS biopsies. Mesangial proliferation was the most common histopathological pattern. Steroid dependency with frequent relapses was the most frequent outcome, with a second line immunosuppressant added, yet without impact on progression. Small sample size hinders coherent conclusions; nevertheless, it indicates that $\mathrm{ClqN}$ is a rare cause of pediatric NS. C1qN may require second line immunosupressants more often than non-Clq NS.

Key words: glomerulonephritis, nephrotic syndrome, C1q nephropathy.

C1q nephropathy $(\mathrm{C} 1 \mathrm{qN})$ is an idiopathic glomerulopathy typically presenting in children and young adults ${ }^{1}$. Patients classically present with nephrotic syndrome (NS). The key diagnostic features are dominant or codominant glomerular mesangial C1q deposition evidenced by immunoflourescence studies (IF), or immunohistochemical stain (IHC) for antiC1q; provided that other immune complex glomerulonephritides like lupus nephritis are excluded by clinical and serologic tests ${ }^{2}$. Large-scale case studies of C1qN are sparse, with controversy regarding the prognosis and proper patient management.

Examination of $\mathrm{Clq}$ deposition by IF or IHCs are currently not performed in our institution, thus, the exact incidence of $\mathrm{ClqN}$ and its prognosis is unknown. We aimed to investigate in retrospect using IHC, the prevalence of $\mathrm{ClqN}$ in the pediatric renal disease population attending renal pediatric services during the period from 2003 to 2016; and to determine the impact of C1qN on disease course and patient outcome especially the risk of progression to chronic renal failure at final follow up, providing clinicopathological correlation that can be implemented for therapeutic purposes.

\section{Material and Methods}

In this retrospective analysis, clinical data were gathered from selected pediatric renal patient's medical papers and electronic records following approval from Faculty of Medicine and University Hospital IRB ethics committee/ report number(KT/14/2014) according to ethical institutional protocols. A total of 105 renal biopsies were found. Gathered data included age, sex, clinical presentation, serum creatinine at presentation and at final follow up, proteinuria at initial diagnosis and at final follow up, histopathological features, IF results, type of therapy, response to treatment, overall follow up period, and final outcome. 
A group of clinical definitions were used to verify the data ${ }^{2}$ : NS is a triad of heavy proteinuria, hypoalbuminemia and edema. Steroid therapy is the mainstay of NS therapy according to the International Study of Kidney Disease in Children (ISKDC) regimen. Initial episodes are usually treated with prednisolone dose of $60 \mathrm{mg} / \mathrm{m}^{2}$ daily for 4 weeks and followed by $40 \mathrm{mg} / \mathrm{m}^{2}$ on alternate days for a further 4 weeks. Nephrotic proteinuria is defined as $\geq 50 \mathrm{mg} / \mathrm{kg} /$ day or $\geq 40 \mathrm{mg} / \mathrm{m}^{2}$ / day. Response to primary (steroid) treatment was classified as either: steroid sensitive (SS): complete remission of proteinuria during the treatment and the remission persisted for at least 2 months after stopping steroids; steroid dependent (SD): is the occurrence of a relapse while the child is still on steroid treatment or within 2 weeks after its cessation; and steroid resistant (SR), defined as no remission over a period of at least 4 consecutive weeks of steroid therapy. Final patient outcome was set according to clinical information and laboratory values are as follows: Remission: $\mathrm{a} \geq 0.15 \mathrm{~g} / 24$ $h$ reduction in urine protein concentration. Relapse was defined as reappearance of proteinuria on at least 3 consecutive days. Frequent relapse (FR) was defined as 2 relapses in 6 months or $\geq 4$ relapses in 12 months.

For the 105 renal biopsies, de-identified, hematoxylin \&eosin-stained microscopic glass slides and the corresponding paraffin-fixed tissue blocks were retrieved from the archives of pathology department. The slides were reviewed by a nephropathologist.

According to a set of diagnostic criteria for $\mathrm{ClqN}$ as determined by Jennette et al. ${ }^{1}$, the diagnosis depends on Clq deposition detected by IHC or IF, after exclusion of immune complex GN like lupus nephritis. A wide spectrum of LM findings were allowed, including: Minimal change disease (MCD), focal and segmental sclerosis pattern (FSGS), mesangial proliferative pattern (MP), and crescentic GN (CGN). Also, as a disease typically presenting with NS, we aimed to investigate incidence among NS cases. Keeping that in mind, selection of cases to perform anti-Clq antibody (anti-Clq) by IHC was done, and included:

a) A clinical presentation of nephrotic proteinuria (N.Pr)/NS;

b) Cases with documented negative or inconclusive previous IF reports that lacked testing for $\mathrm{Clq}$.

c) Exclusion of cases with diagnostic features of other immune complex glomerulonephritides (like lupus nephritis (LN), IgA nephropathy, membranous nephropathy, etc) by clinical and serologic tests.

Three samples were initially excluded for being inadequate for evaluation. Categorization of cases $(n=102)$ was then performed as previously mentioned into Group 1(criteriapositive; $\mathrm{n}=59$ ) and Group 2 (criteria-negative; $n=43$ ). In Group 1, six tissue blocks were excluded because they were either not found or considered destitute to perform IHC. Accordingly, a total of 53 biopsies (Group 1) were selected to perform the anti-Clq IHC staining as described below.

IHC stains were performed manually on the corresponding tissue blocks, using AntiC1qA antibody [clone: EPR2980Y] Rabbit IgG monoclonal concentrated antibody (Gennova/ Spain). For the formalin-fixed, paraffinembedded tissue, a concentration of $1 \mu \mathrm{g} / \mathrm{ml}$ was used. Heat-mediated antigen retrieval was done before commencing with IHC protocol. Positive and negative tissue controls were run with each set of slides. The results were interpreted by a nephropathologist using a LM, including the presence or absence of glomerular stain, stain intensity, and location within the glomeruli.

Clinicopathological correlation was performed using the LM findings, Clq deposition, and the clinical parameters of the corresponding cases, with center of attention on the mode of therapy, response to treatment, disease progression and outcome. Descriptive statistics of mean was used for continuous variables, and numbers (percentages) for categorical variables.

\section{Results}

\section{Diagnostic categories of renal biopsies among pediatric cases}

The pediatric renal biopsies included 102 native kidney biyopsies and 3 kidney transplant biopsies. The patients were 51 females and 54 males (ratio of $0.95: 1$ ). The mean age was 7.4 years (ages ranged from 3 months to 18 years). Clinical follow up (FU) was available for 96 cases. The follow up periods 
Table I. Clinical Data of Group 1 (C1q+ versus C1q-).

\begin{tabular}{|c|c|c|}
\hline \multirow[t]{2}{*}{ Criteria } & \multicolumn{2}{|c|}{ Group1 (criteria+) } \\
\hline & $\mathrm{Clq}+$ & $\mathrm{Clq}^{-}$ \\
\hline Number of patients & 5 & 48 \\
\hline Patients with follow-up & 4 & 43 \\
\hline$\%$ of Group 1 & $9.4 \%$ & $90.6 \%$ \\
\hline$\%$ of all samples & $4.9 \%$ & $47.1 \%$ \\
\hline Initial creatinine $($ mean $\pm S D)$ & $0.4 \pm 0.23 \mathrm{mg} / \mathrm{dl}$ & $0.47 \pm 0.75 \mathrm{mg} / \mathrm{dl}$ \\
\hline Initial creatinine $($ mean $\pm S D)$ & $0.28 \pm 0.15 \mathrm{mg} / \mathrm{dl}$ & $1.8 \pm 2.5 \mathrm{mg} / \mathrm{dl}$ \\
\hline Initial urinary protein $($ mean $\pm S D)$ & $1.89 \pm 1.26 \mathrm{~g} /$ day & $1.47 \pm 2.3 \mathrm{~g} /$ day \\
\hline Initial urinary protein $($ mean $\pm S D)$ & $2.6 \pm 2.3 \mathrm{~g} /$ day & $1.92 \pm 2.46 \mathrm{~g} /$ day \\
\hline Mean follow-up & 42 month & 36.69months \\
\hline Treatment & $+/-15.49$ & +/- 39.79 \\
\hline Number of patients & 5 & 43 \\
\hline Steroid sensitive & $0(0 \%)$ & $21(48.8 \%)$ \\
\hline Steroid dependent & $0(0 \%)$ & $7(16.3 \%)$ \\
\hline Steroid dependent $+2^{\text {nd }}$ drug & $3(60 \%)$ & $6(14 \%)$ \\
\hline Steroid resistant & $1(20 \%)$ & $4(9.3 \%)$ \\
\hline No treatment & $1(20 \%)$ & $5(11.6 \%)$ \\
\hline \multicolumn{3}{|l|}{ Outcome } \\
\hline Alive at FFU & 4 & 37 \\
\hline Remission & $1(20 \%)$ & $21(48.8 \%)$ \\
\hline Frequent relapse & $3(60 \%)$ & $6(14 \%)$ \\
\hline Persistent proteinuria & $1(20 \%)$ & $7(16.2 \%)$ \\
\hline ESRD & $0(0 \%)$ & $3(7 \%)$ \\
\hline Deaths* & $1(20 \%)^{*}$ & $6(14 \%)$ \\
\hline
\end{tabular}

SD: standard deviation; FUP: follow up period; FFU: final follow up; ESRD: end stage renal disease; ${ }^{*}$ refers to death due to ESRD

(FUP) ranged from 1 to 156 months (mean= $38.56 \mathrm{mo})$. According to clinical presentation and initial laboratory work up, the most common presentation was nephrotic-range proteinuria (N.Pr) in 59 patients $(57.9 \%)$, 39 others had non-nephrotic proteinuria, and 18 had no proteinuria. Ten patients initially presented with renal failure $(9.5 \%$ of all). Initial serum creatinine $(\mathrm{Cr})$ values ranged from 0.09 to $5.78 \mathrm{mg} / \mathrm{dl}(0.66 \pm 0.96)$. Cr readings at final follow up (FFU) ranged from 0.04 to $13.50 \mathrm{mg} / \mathrm{dl}(1.56 \pm 2.55)$. Initial urine tests showed no hematuria in 83 cases, microscopic hematuria in 6 , and macroscopic hematuria in 13 patients. 3 patients presented initially with hypertension (HTN). Two patients had renal anatomical defects (posterior urethral valves) in addition to nephrotic range proteinuria.
Among the 59 cases selected for the current study, 6 were excluded since the tissue blocks were not found or considered meager for staining $(n=59-6=53)$. Out of the 53 cases, 40 cases had a histopathological diagnosis of MP $(75.5 \%), 8$ had FSGS (15.1\%), 7 had MCD (13.2\%), with one had NS post renal allograft, 2 cases of congenital nephrotic syndrome, 2 cases with crescentic GN.FU of biopsied NS cases showed the most common outcome to be remission $(41 \%)$. Persistent proteinuria was observed in $18 \%$, and $16 \%$ eventually progressed to ESRD. Clinical data of cases with and without C1q detection are summarized in Table I.

Results of IHC with anti-C1q: Group 1 a $(\mathrm{Clq}+)$ vs. group $1 b$ (C1q-) 
Out of the 53 anti-Clq -tested tissue blocks, 5 blocks ( $9.4 \%$ of stained cases) displayed C1q deposition. The deposition was moderate intensity and mesangial in location in all cases. Light microscopic findings are displayed in Figure 1. One paraffin-fixed tissue block for one Clq-positive sample was de-parafinized for electron microscopic (EM) examination by transmission electron microscope, following retrieval procedures, and the glomeruli showed mesangial immune deposits, as shown in Figure 2. Due to the small number of samples that were $\mathrm{Clq}+$, statistical analysis was not justified; however, the clinicopathological features of those cases are detailed below.

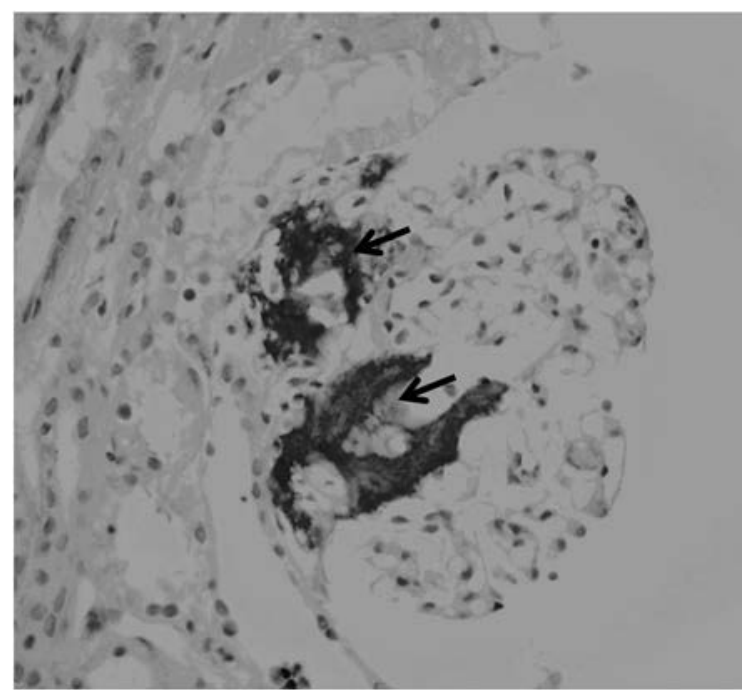

Fig. 1. Light microscopic findings in $\mathrm{ClqN}$. IHC stain with anti-Clq showing a glomerulus with mesangial $\mathrm{Clq}$ deposition (arrows) as well as in segmental sclerosis. (original magnification 100X).

\section{Clinicopathological features of $\mathrm{C} 1 q+$ cases}

Our case series included 5 patients $(n=5)$. The patient ages ranged from 2 to 12 years (mean $=7.4$ years). They were 4 males and 1 female, clinically presented with NS. Urinary protein values at FFU were nil in one patient and $3+$ (heavy) in the other three. Initial $\mathrm{Cr}$ values ranged from 0.13 to $0.7 \mathrm{mg} / \mathrm{dl}$. Cr values at FFU were similar, ranging from 0.13 to 0.45 .

The FUP ranged from 24 to 60 mo. (mean= 44 mo.). One (patient 1) was lost to follow up (LFU). Patient 2 had a relapse 4 year following initial biopsy, yet is doing well now, with $\mathrm{Cr}$ of 0.45 and no proteinuria. The remaining cases (patients 3, 4 and 5) were NS-SD; with FR; they had an immunosuppressant drug added as a second line of therapy (cyclophosphamide and cyclosporine). Except for patient 1 who was LFU and unfortunately found to be dead due to ESRD, none of the cases with FU showed progression to ESRD. Examination of the microscopic slides revealed MP pattern in all cases. Review of the old IF reports that did not include testing for Clq deposition, was negative for immunoglobulins and complements in 2 cases, one case showed mesangial IgM 1+, and trace IgA, while the IF was not performed in 1 case. In this study, all five cases showed mesangial location of $\mathrm{Clq}$ deposits, with weak to moderate intensity.

\section{Patient 1}

A 12-year-old boy, presenting with edema and N.Pr, was initiated on prednisolone therapy but did not complete his therapy course and was discharged from hospital by family against
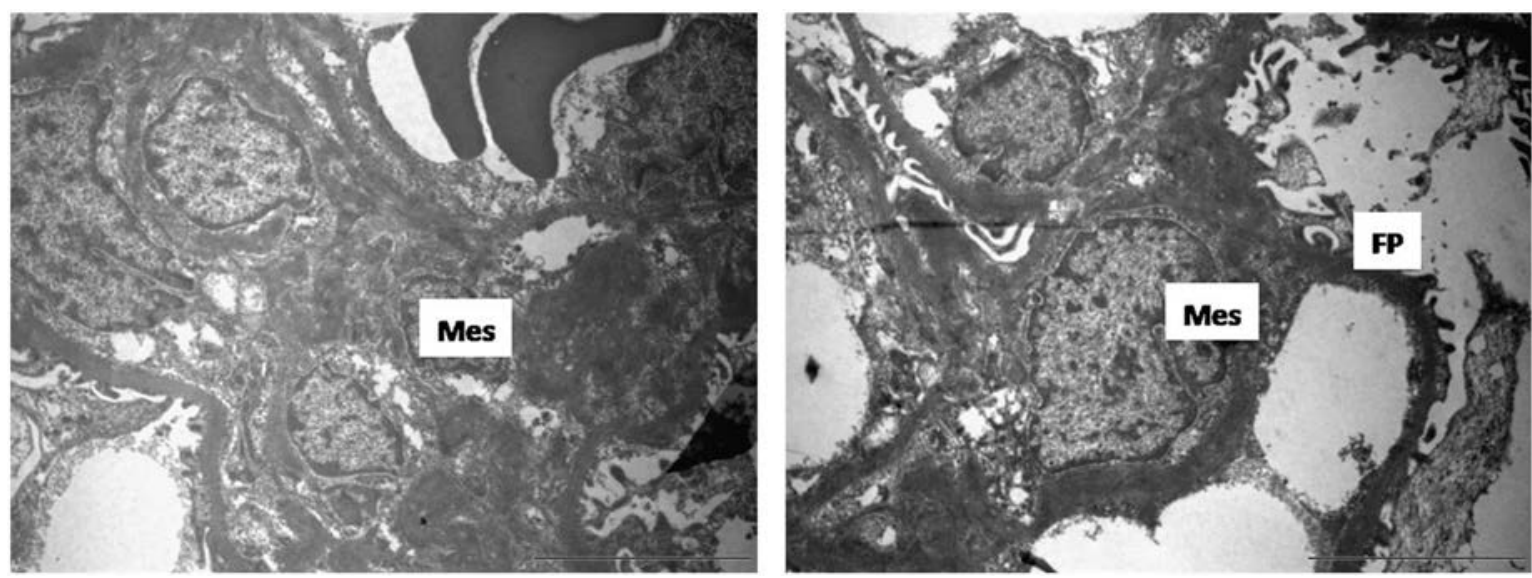

Fig. 2. Ultrastructural features of $\mathrm{ClqN}$. Electron microscopic examination reveals mesangial (Mes) expansion by electron dense deposits, and adjacent podocyte foot process (FP) effacement. (Transmission Electron Microscope, 8,000X). 
medical advice. Unfortunately, he was LFU and upon medical archive retrieval was found to be dead due to renal failure.

\section{Patient 2}

The patient is a 6-year-old boy, who presented to pediatric clinics with NPr. He was treated with steroids and his clinical course was significant for SD. Second line drug (cyclosporine) was added after 2 yrs. He had his last relapse on June 2008 after 4 years of initial diagnosis. At final FU, he was found well with no proteinuria.

\section{Patient 3}

A 5-year-old male presented with NS/SD, his serologic work up showed normal serum C3. His disease course was significant for FR. Cyclosporine as a second line drug was added after 3 yrs on Nov, 2008. He was last seen on Dec, 2009, when he was doing well 1 year after last relapse.

\section{Patient 4}

A 2-year-old female with NS/SD, started on steroids initially, then cyclophosphamide was added 6 months later. She was still symptomatic at her final follow up, when she started to develop resistance to both steroids and cyclophosphamide. She also complained of precocious puberty 2 years since her initial diagnosis.

\section{Patient 5}

A 12-year-old male, presenting with $3+$ urine dipstick protein, was evaluated and treated with steroids. An outside electron microscopic report for his kidney biopsy was suspicious of Fabry's disease, which is a lysosomal storage disease that frequently leads to proteinuria and progression to renal failure by adulthood ${ }^{3}$. During the FUP of 48 months his clinical course was significant for many admissions, NS- SD, and had FR. Cyclosporine was added 24 months of initial diagnosis as a steroid sparing agent.

\section{Discussion}

$\mathrm{C} 1$ is a complement component of the classical complement pathway. $\mathrm{C} 1 \mathrm{q}$ plays a role in several immunological processes, like triggering of bacterial phagocytosis, neutralization of retroviruses and safeguarding of immune tolerance via the removal of apoptotic cells ${ }^{4}$. $\mathrm{C} 1 \mathrm{q}$ receptors are expressed on different cell types, including leukocytes, fibroblasts, platelets, endothelial cells and renal mesangial cells ${ }^{4}$. Furthermore, auto-antibodies against $\mathrm{Clq}$ have been found in a number of autoimmune diseases, specifically $\mathrm{LN}^{5}$, were they have a high negative predictive value in the detection of active disease. ${ }^{5}$ Knowledge of pathogenesis of immune-mediated diseases will help in development of targeted therapy to block complement activation. ${ }^{6}$

$\mathrm{ClqN}$ is an uncommon entity, where Clqcontaining immune deposits are detected in the mesangium. It is considered an immune complex-mediated glomerulonephritis. The term was initially proposed by Jennette and Hipp $^{1}$ in 1985. Pathophysiology of ClqN had been an issue of controversy for the past 30 years, mostly regarding whether $\mathrm{ClqN}$ is a single disease, or a mixture of several diseases. Many researchers think of $\mathrm{ClqN}$ as subgroup of primary $\mathrm{FSGS}^{7}$. However, many others proposed it as a separate disorder as evidenced by different clinical, histopathological features, therapeutic responses and prognoses. ${ }^{8}$ There are other uncertainties about $\mathrm{ClqN}$ that require further investigations. First, detection of Clq deposition in other types of GN, like LN. However, $\mathrm{LN}$ can be distinguished from $\mathrm{ClqN}^{4}$, on clinical and serologic backgrounds, and by the fact that $\mathrm{Clq}$ deposition is accompanied by a full-house pattern of immunoglobulin deposition. ${ }^{5}$ Therefore, ClqN should be only diagnosed after exclusion of various types of immune complex-mediated GN. ${ }^{8}$ Second, is the clinical and microscopic heterogeneity, as detailed below:

From a histopathological perspective, this disorder refers to $\mathrm{Clq}$ deposition escorted by glomerular injury with a broad range of potential findings, including MCD-like disease, FSGS, and MP pattern. The diagnostic criteria of ClqN were set in 1985 by Jennette et al. ${ }^{1}$, and those include: (1) dominant or codominant mesangial IF staining for Clq; (2) mesangial electron-dense deposits by EM; and (3) and absence of clinical and serologic (antinuclear antibodies) evidence of LN.It has been disputed that the LM heterogeneity in $\mathrm{ClqN}$ is akin to that of IgA nephropathy, and thus the diagnosis should be determined by C1q staining 1,9 either by IHCs or IF. However, several points should be excluded first: the presence of another immune-complex disease 


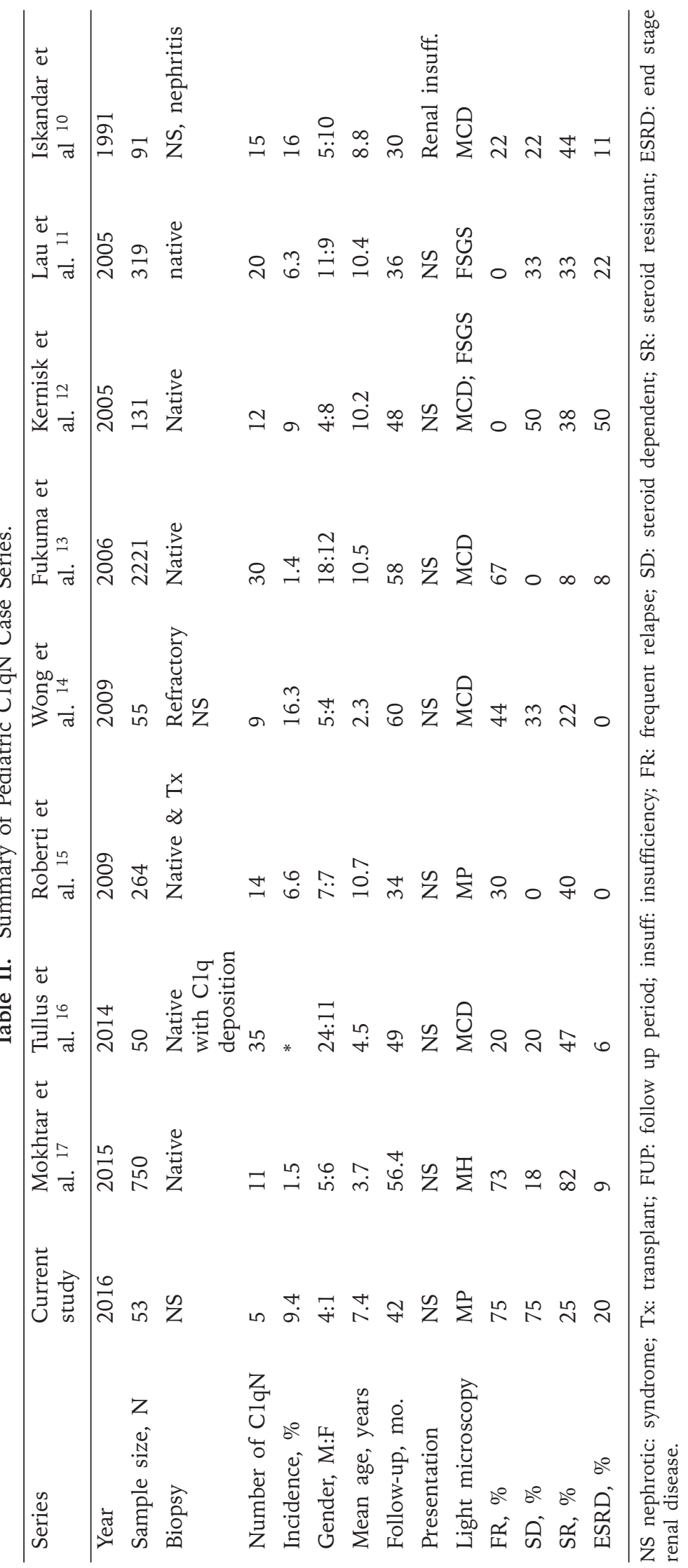


associated with co-deposition of C1q, like LN, IgA, or IgM nephropathy; and second, the false positive results of $\mathrm{Clq}$ trapping in sclerosed segments or globally sclerosed glomeruli, as previously noted. ${ }^{7}$

Due to the recent awareness of $\mathrm{ClqN}$ and its rarity, the number of comprehensive large-scale studies on $\mathrm{ClqN}$ had been modest. Besides some case reports and small adult case series, there had been eight exclusively pediatric case series of $\mathrm{ClqN} .^{9-17}$ Major clinicopathological parameters of those pediatric case series are summarized in Table II.

Generally, the incidence of $\mathrm{ClqN}$ shows a broad range among different pediatric and adult studies, ranging from as low as $0.2 \%$ to as high as $16.5 \%$ in pediatric cases with persistent NS. ${ }^{6}, 12$ Our study had an incidence of $9.4 \%$ among pediatric NS cases. The wide spectrum of incidence among the different studies is due to the study population, as it is much higher in NS patients compared to random studies on general biopsy populations. Another recent study from Middle East, focusing on SR-NS patients, had showed that the incidence of ClqN was about $8 \% .{ }^{18}$ The mean age among those studies ranges from as young as 2.3 to 10.7 yrs. The gender distribution had moved back and forth in favor of male gender in 4 previous studies, while it had a female predominance in 3 others, and showed equal incidence in one other study.

The optimal management of $\mathrm{C} 1 \mathrm{qN}$ is not clearly defined yet. Corticosteroids have been used as a first line with diverse results, with some studies showing poor response to oral corticosteroid therapy ${ }^{10}$, while others shows superior results. ${ }^{14}$

There had been substantial disagreement over the outcome of patients with ClqN.

As it had been previously described, the outcomes of patients with $\mathrm{ClqN}$ can be predicted by an array of clinical and histopathological variables. ${ }^{6}$ Precautious evaluation of the previous pediatric case series makes it clear that the dominant LM pattern had a higher impact in predicting disease progression and outcome at FFU, even regardless of $\mathrm{C} 1 \mathrm{q}$ deposition. ${ }^{8}$ Tulluset al. ${ }^{16}$ had confirmed that cases presenting with a LM picture of MCD had better general outcome compared to those with FSGS, or MP. They also show better response to steroids, although not statistically significant. ${ }^{16}$ Nevertheless, patients with MCD/Clq deposition seemed to need second-line drugs as cyclosporine despite having comparable renal outcome at FFU.14

Regarding overall disease progression to ESRD, some studies failed to show any statistical significance distinguishing cases with $\mathrm{Clq}$ from non-C1q patterns, and both patient groups exhibited an analogous outline. ${ }^{16}$

In conclusion, our current study had comparable results to previous pediatric case series in clinical and histologic parameters. MP was the most common LM pattern. All cases presented clinically as NS. SD-NS was the most frequent outcome, however, with no statistical impact on progression to renal failure. The small number in our study hinders accurate statistical assurance, however, our findings also point towards a superior role of LM pattern over $\mathrm{C} 1 \mathrm{q}$ deposits regarding disease progression. It may, also signify need of second line drugs in ClqN. Larger studies are needed to establish solid management guidelines of $\mathrm{ClqN}$.

\section{Acknowledgement}

We are thankful for Dr. Khalid Wesam, and Dr. Fatin Al-Fares (Department of Pathology) for medical records retrieval.

\section{REFERENCES}

1. Jennette JC, Hipp CG. Clq nephropathy: a distinct pathologic entity usually causing nephrotic syndrome. Am J Kidney Dis 1985; 6: 103-110.

2. Zolotas E, Krishnan RG, Nephrotic syndrome. Pediatrics and Child Health 2016; 26: 349-352.

3. Ortiz A, Cianciaruso B ,Cizmarik M, et al. End-stage renal disease in patients with Fabry disease: natural history data from the Fabry Registry. Nephrol Dial Transplant 2010; 25: 769-775.

4. Prodinger WM WrR, Stoiber H, Dierich MP. Complement. In: Paul W E (ed). Fundamental immunology (5th ed) Vol.1.Philadelphia: Lippincott, Williams \&Wilkins, 2003: 1077-1103.

5. Potlukova E, Kralikova P. Complement component $\mathrm{Clq}$ and anti-Clq antibodies in theory and in clinical practice. Scand J Immunol 2008; 67: 423-430.

6. Malleshappa P, Vankalakunti M. Diverse clinical and histology presentation in clq nephropathy. Nephrourol Mon 2013; 5: 787-791.

7. Markowitz GS, Schwimmer JA, Stokes MB, et al. Clq nephropathy: A variant of focal segmental glomerulosclerosis. Kidney Int 2003; 64: 1232-1240. 
8. Mii A, Shimizu A, Masuda Y, et al. Current status and issues of C1q nephropathy. Clin Exp Nephrol 2009; 13: 263-274.

9. Wenderfer SE, Swinford RD, Braun MC. C1q nephropathy in the pediatric population: Pathology and pathogenesis. Pediatr Nephrol 2010; 25: 13851396.

10. Iskandar SS, Browning MC, Lorentz WB. C1q nephropathy: A pediatric clinicopathologic study. Am J Kidney Dis 1991; 18: 459-465.

11. Lau KK, Gaber LW, Delos Santos NM, Wyatt RJ. C1q nephropathy: Features at presentation and outcome. Pediatr Nephrol 2005; 20: 744-749.

12. Kersnik Levart T, Kenda RB, Avgustin Cavic M, Ferluga D, Hvala A, Vizjak A. C1q nephropathy in children. Pediatr Nephrol 2005; 20: 1756-1761.

13. Fukuma Y, Hisano S, Segawa Y, et al. Clinicopathologic correlation of $\mathrm{Clq}$ nephropathy in children. Am J Kidney Dis 2006; 47: 412-418.
14. Wong CS, Fink CA, Baechle J, Harris AA, Staples AO, Brandt JR. Clq nephropathy and minimal change nephrotic syndrome. Pediatr Nephrol 2009; 24: 761767.

15. Roberti I, Baqi N, Vyas S, Kim DU. A single-center study of Clq nephropathy in children. Pediatr Nephrol 2009; 24: 77-82.

16. Gunasekara VN, Sebire NJ, Tullus K. Clq nephropathy in children: Clinical characteristics and outcome. Pediatr Nephrol 2014; 29: 407-413.

17. Mokhtar GA, Jalalah SM. A clinicopathological study of Clq nephropathy at King Abdulaziz University. Iran J Kidney Dis 2015; 9: 279-285.

18. Kari JA, Halawani M, Mokhtar G, Jalalah SM, Anshasi W. Pattern of steroid resistant nephrotic syndrome in children living in the kingdom of Saudi Arabia: a single center study. Saudi J Kidney Dis Transpl 2009; 20: $854-857$. 